\title{
Efecto del faique (Acacia macracantha) sobre el valor nutricional del pasto guinea (Panicum maximum Jacq.) en un sistema silvopastoril
}

\author{
Effect of the faique (Acacia macracantha) on the nutritional value of guinea grass \\ (Panicum maximum Jacq.) in a silvopastoral system \\ Gelver Romero Delgado, Mariano Echevarría Rojas² ${ }^{2}$ Fritz Trillo Zárate ${ }^{1}$, Víctor \\ Hidalgo Lozano ${ }^{2}$, Lucrecia Aguirre Terrazas ${ }^{1}$, Rafael Robles Rodríguez ${ }^{3}$, Jimny \\ Núñez Delgado ${ }^{1,4}$,
}

\section{Resumen}

\begin{abstract}
El objetivo del estudio fue determinar la tasa de crecimiento y calidad nutricional del pasto Guinea (Panicum maximum Jacq) bajo un sistema silvopastoril con el árbol faique (Acacia macracantha). Se evaluaron dos épocas (lluviosa y seca) y tres tratamientos: (1) bajo la copa del árbol joven, (2) bajo la copa del árbol mediano y (3) fuera de la copa del árbol. Los árboles se clasificaron según el diámetro a la altura al pecho. El crecimiento y calidad del pasto se midió por el método de corte y separación y posterior análisis de laboratorio. La tasa de crecimiento osciló de 71.09 a $51.15 \mathrm{~kg} \mathrm{MS} / \mathrm{ha} / \mathrm{d}$, fuera y dentro de la copa de los árboles, respectivamente, en la época lluviosa. La proteína cruda varió de $10.58,12.79$ y $13.88 \%$ (época lluviosa) y $8.93,10.26$ y $11.99 \%$ (época seca) fuera de la copa del árbol, bajo la copa del árbol joven y bajo la copa del árbol mediano, respectivamente $(\mathrm{p}<0.05)$. El contenido de calcio en el pasto fue estadísticamente $(\mathrm{p}<0.05)$ superior bajo la copa del árbol mediano ( $0.23 \%)$ que fuera de la copa del árbol $(0.20 \%)$ en la época lluviosa. El fósforo en el pasto varió significativamente $(\mathrm{p}<0.05)$ entre $0.72,0.83$ y $0.91 \%$ (época lluviosa) y $0.73,0.87$ y $1.06 \%$ (época seca), fuera de la copa de los árboles, bajo la copa del árbol joven y bajo la copa del árbol mediano, respectivamente. El estudio reveló que la presencia de árboles leguminosos (Acacia macracantha) dentro de los potreros mejora la calidad nutricional de la pastura Panicum maximum Jacq.
\end{abstract}

Palabras clave: calidad nutricional, crecimiento, copa del árbol, pastura, sistema silvopastoril

\footnotetext{
${ }^{1}$ Laboratorio de Ecología y Utilización de Pastizales, Facultad de Zootecnia, Universidad Nacional Agraria La Molina, Lima, Perú

${ }^{2}$ Departamento de Nutrición, Facultad de Zootecnia, Universidad Nacional Agraria La Molina, Lima, Perú

${ }^{3}$ Departamento de Ciencias Pecuarias, Universidad Nacional Agraria de la Selva, Tingo María, Perú

${ }^{4}$ E-mail: jimny_unalm@hotmail.com
}

Recibido: 18 de febrero de 2019

Aceptado para publicación: 18 de enero de 2020

Publicado: 31 de marzo de 2020 
The aim of this study was to determine the growth rate and nutritional quality of Guinea grass (Panicum maximum Jacq) under a silvopastoral system with the faique tree (Acacia macracantha). The evaluation included two seasons (rainy and dry) and three treatments: (1) under the canopy of the young tree, (2) under the canopy of the mediumsize tree, and (3) outside the canopy of the tree. The trees were classified according to the diameter at chest height. The growth and quality of the grass were measured by the method of cutting and separation and subsequent laboratory analysis. The growth rate ranged from 71.09 to $51.15 \mathrm{~kg} \mathrm{DM} / \mathrm{ha} / \mathrm{d}$, outside and inside the tree canopy, respectively, in the rainy season. Crude protein varied from $10.58,12.79$ and $13.88 \%$ (rainy season) and $8.93,10.26$ and $11.99 \%$ (dry season) outside the tree canopy, under the young tree canopy and under the middle tree canopy respectively $(\mathrm{p}<0.05)$. The calcium content in the grass was statistically $(\mathrm{p}<0.05)$ higher under the middle tree canopy $(0.23 \%)$ than outside the tree canopy $(0.20 \%)$ in the rainy season. The phosphorus in the grass varied significantly $(\mathrm{p}<0.05)$ between $0.72,0.83$ and $0.91 \%$ (rainy season) and $0.73,0.87$ and $1.06 \%$ (dry season), outside the tree canopy, under the canopy of the young tree and under the canopy of the medium tree, respectively. The study revealed that the presence of leguminous trees (Acacia macracantha) within the paddocks improves the nutritional quality of the Panicum maximum Jacq pasture.

Key words: nutritional quality, growth, tree crown, pasture, silvopastoral system

\section{INTRODUCCIÓN}

Los sistemas ganaderos tropicales en el Perú se caracterizan por ser silvopastoriles, combinando pasto, árboles y animales. La presencia de árboles dentro de los potreros, cumplen un rol importante en el sistema, brindando sombra y forraje para los animales, además de ser un componente importante al estar en simbiosis con el pasto. El pasto guinea (Panicum maximum Jacq) es la especie naturalizada predominante en ecosistemas de selva alta (Nuñez, 2017). Así mismo, en dichas áreas es frecuente la deforestación, tala y quema indiscriminada de los bosques para el establecimiento de pasturas, lo cual trae una disminución de la calidad, productividad y diversidad de la vegetación (Oliva, 2016).

El uso se especies arbóreas en los sistemas silvopastoriles (SSP) ofrece ventajas para el desarrollo de ganaderías con menor impacto ambiental. Una de las bondades de
SSP es la obtención de una mejor calidad y cantidad de pasto (Obispo et al., 2008), permitiendo un mayor rendimiento en los sistemas ganaderos (FAO, 2012). Los SSP presentan también un gran potencial para recuperación de áreas de pasturas degradadas (Alegre et al., 2012); sin embargo, se requiere determinar las interacciones entre sus componentes para asegurar el correcto funcionamiento del sistema.

Panicum maximum es una especie tolerante al pisoteo y la sequía, y es un alto productor de forraje de buena calidad, palatabilidad y digestibilidad; desarrollándose muy bien en SSP con una clara ventaja sobre otras especies de pasto, porque tiene la capacidad de soportar ciertos niveles de sombra sin interferir en su producción (Ledesma, 2006). No obstante, su rendimiento se ve afectado por el régimen de lluvias, mostrando niveles inferiores en épocas secas (Núñez, 2017). Por otro lado, se tiene evidencia de especies de pasto que crecen adecuadamen- 
te bajo la sombra de otras plantas (Abraham et al., 2014).

El valor nutritivo de la planta declina a medida que avanza en edad debido a un incremento en su lignificación, aumento de la fibra detergente neutro y menor contenido de proteína, así como una disminución del número de hojas (García, 2002). Así mismo, la sombra también puede provocar cambios morfológicos y fenológicos en la especie forrajera, los cuales funcionan como mecanismo de adaptación a la baja incidencia de energía lumínica y la consiguiente reducción en el potencial fotosintético de la planta. Para compensar esto, la especie forrajera tiende a desarrollar hojas largas, pero menos gruesas para una mejor captación de luz y una menor tasa de respiración (Wilson y Ludlow, 1991).

No existen estudios sobre los beneficios de los árboles como el faique (Acacia macracantha) dentro de los potreros en praderas tropicales, de allí que el objetivo del estudio fue determinar las tasas de crecimiento y el valor nutricional del pasto bajo la copa de los árboles y fuera de ella en un sistema silvopastoril, donde el componente arbóreo fue el faique y el componente forrajero fue el pasto guinea (Panicum maximum Jacq.) para determinar su importancia dentro del sistema de producción pecuario de la zona de Cajamarca, Perú.

\section{MATERIALES y Métodos}

\section{Localización}

La fase experimental se realizó en la región de selva alta (2016), distrito de Bellavista, provincia de Jaén, Región Cajamarca (Perú), a 729 msnm. La zona está clasificada de acuerdo con Holdridge (2000) como bosque seco premontano tropical (bs-PT). La temperatura media anual es de $24.2{ }^{\circ} \mathrm{C}$ con máximas y mínimas promedio de $34.0 \mathrm{y}$ $18.5{ }^{\circ} \mathrm{C}$, respectivamente. La precipitación anual es $810 \mathrm{~mm}$, siendo la mayor en marzo
(197 mm) y la menor en noviembre (16 mm) (SENAMHI, 2018). El componente pasto contempló 8 ha de Panicum maximum Jacq. (monocultivo naturalizado), establecido hace 20 años, en asociación con la especie arbórea leguminosa nativa Acacia macracantha, conocida comúnmente como faique.

El sistema es manejado en secano ( $\sin$ riego) con sistemas de pastoreo rotativo cada seis semanas. La pastura residual tuvo una altura promedio de 60 y $20 \mathrm{~cm}$ en época lluviosa y seca, respectivamente. El componente animal estuvo representado por ganado vacuno criollo de doble propósito. La evaluación se realizó en la época lluviosa (marzo) y seca (agosto) de 2017.

\section{Análisis de Suelo}

El muestreo de suelo (6 muestras en total) se hizo a $30 \mathrm{~cm}$ de distancia del punto de colección de las muestras de pasto, pero a 25 a $30 \mathrm{~cm}$ de profundidad, que es donde se encuentra la mayor proporción del sistema radicular del Panicum maximum Jacq. La humedad del suelo se midió por gravimetría (Martínez, 1971). Las muestras consistieron en $1 \mathrm{~kg}$ de suelo utilizando envases con cierre hermético para evitar la pérdida de humedad, y luego fueron enviadas al Laboratorio de Suelos, Plantas, Agua y Fertilizantes de la Universidad Nacional Agraria la Molina, Lima donde se colocaron en estufa a $105^{\circ} \mathrm{C}$ durante $24 \mathrm{~h}$. La temperatura del suelo se midió dentro del excluidor de crecimiento del pasto, a una profundidad de 15 a $20 \mathrm{~cm}$ de la superficie, mediante un geotermómetro (Reotem ${ }^{\circledR}$, rango: -10 hasta $90{ }^{\circ} \mathrm{C}$, con $0.5^{\circ} \mathrm{C}$ de precisión) por 5 minutos.

\section{Categorización de los Árboles}

La densidad promedio fue de 80 árboles dispersos por hectárea. La categorización del tamaño de los árboles se hizo mediante la medición del Diámetros a la Altura del Pecho (DAP), según la FAO (2004), utilizando la siguiente ecuación: $\mathrm{D}=\mathrm{C} / \pi$, donde $\mathrm{D}$ : diámetro $(\mathrm{cm})$, C: circunferencia $(\mathrm{cm}), \pi: 3.1416$. 
Para ello se utilizó una cinta métrica para medir la circunferencia (C) a la altura del pecho (1.3 m).

Las categorías del tamaño de los árboles por hectárea fueron: árboles en regeneración (DAP $<7.5 \mathrm{~cm}$ ) $13.4 \%$, jóvenes (DAP 7.5-24 cm) 50.6\%, pequeños (DAP 25-37 cm) $17.3 \%$ y medianos (DAP $38-49 \mathrm{~cm}$ ) $18.5 \%$. Los muestreos de pasto y suelo se realizaron en la cuarta semana de rebrote del pasto, en época lluviosa (enero-marzo) y seca (julio-setiembre).

\section{Crecimiento del Pasto}

Se utilizaron cuatro potreros de 2 ha cada uno. En cada portero se instalaron tres excluidores $\left(4 \mathrm{~m}^{2}\right)$ a base de alambre de púas para evitar el pastoreo, distribuidas de la siguiente manera: fuera la copa del árbol (FCA), bajo la copa del árbol joven (BCAj) y bajo la copa del árbol mediano (BCAm). El área de muestreo fue de $1 \mathrm{~m}^{2}$ para eliminar el efecto del borde (ASRM, 1962). La tasa de crecimiento (método de corte y separación; Geenty y Rattray, 1987) fue el forraje disponible dentro del excluidor un mes después del corte (10 cm del nivel del suelo), expresado en kilogramos de forraje seco por hectárea por día ( $\mathrm{kg} \mathrm{MS} / \mathrm{ha} / \mathrm{d})$. Para el pesaje se utilizó una balanza digital OHAUS ${ }^{\circledR}$, con capacidad de $3000 \mathrm{~g}$ y precisión de $0.1 \mathrm{~g}$.

\section{Valor Nutritivo del Pasto}

Se colectaron 12 muestras de pasto bajo la copa de los árboles y fuera de ellos. Se muestreó a tres distancias: 1, 2, 4 m del tronco de los árboles. Las muestras fueron secadas a $60^{\circ} \mathrm{C}$ durante 48 horas, y luego fueron pasadas por el molino (Wiley ${ }^{\circledR}$ ) con tamiz de $1 \mathrm{~mm}$. Se determinó: proteína cruda (PC) por el método Kjeldahl (AOAC, 2012), fibra detergente neutro (FDN) por el método Van Soest (Van Soest, 1985), digestibilidad in vitro de la materia seca (DIVMS) por el método de Tilley y Terry (1963), calcio y fósforo por el método digestión ácida (AOAC, 2016).

\section{Análisis Estadístico}

El análisis de variancia de los valores contenidos de tasa de crecimiento, PC, FDN, calcio, fósforo y DIVMS se hizo bajo un diseño de bloques completamente al azar con arreglo factorial $3 \times 2$, donde los bloques fueron los potreros, los factores fueron las épocas del año y la ubicación del pasto. Para la evaluación de comparaciones individuales de los efectos se utilizó la prueba de Duncan a un nivel de significancia de 0.05 . Se utilizó el paquete estadístico $\mathrm{R}$.

\section{Resultados y Discusión}

\section{Características del Suelo}

El Cuadro 1 indica que los suelos del área experimental eran moderadamente alcalinos con valores de $\mathrm{pH}$ entre 8.01 y 8.23 para las tres áreas de medición, siendo ligeramente superior a los valores reportados por Núñez (2017) en la misma zona, pero sin considerar la presencia de árboles. La textura del suelo fue la misma para las tres áreas evaluadas con lecturas Franco-Arcillo-Arenoso, lo que demuestra que son suelos de textura moderadamente fina, buena aireación y disponibilidad de agua, ayudando a los microorganismos en el suelo a sobrevivir, beneficiando la absorción de nutrientes y favoreciendo el desarrollo de las plantas (Navarro, 2013). La cantidad de materia orgánica en el suelo aumenta bajo la copa de los árboles, debido a que esta especie de árbol es de tipo caducifolia (reciclaje de hojarasca).

Los niveles de fósforo y calcio en el suelo bajo la copa de los arboles fueron mayores (Cuadro 1), debido a que la raíces de los arboles tiende a extraer minerales de capas más profundas del suelo, efecto de bombeo de nutrientes (Sadeghian et al., 1998). Similar tendencia mostró el potasio. No se detectaron niveles de aluminio en los suelos debido a que son suelos ligeramente alcalinos. 
Cuadro 1. Características del suelo alrededor de árboles de faique (Acacia macracantha)

\begin{tabular}{llll}
\hline Indicador & FCA & BCAj & BCAm \\
\hline $\mathrm{pH}$ & 8.23 & 8.18 & 8.01 \\
Textura & $\mathrm{Fr}, \mathrm{Ar}, \mathrm{A}$ & $\mathrm{Fr}, \mathrm{Ar}, \mathrm{A}$ & $\mathrm{Fr}, \mathrm{Ar}, \mathrm{A}$ \\
$\mathrm{MO}(\%)$ & 2.81 & 3.62 & 3.73 \\
$\mathrm{CaCO}_{3}(\%)$ & 13.95 & 20.50 & 19.77 \\
$\mathrm{P}(\mathrm{ppm})$ & 6.35 & 10.95 & 9.4 \\
$\mathrm{~K}(\mathrm{ppm})$ & 339.25 & 278.5 & 607.5 \\
$\mathrm{Al}^{+3} \mathrm{H}+$ & 0 & 0 & 0 \\
\hline
\end{tabular}

Fuera de la copa del árbol (FCA), bajo la copa del árbol joven (BCAj), bajo la copa del árbol mediano (BCAm)

Materia orgánica (MO)

\section{Variables de Suelo}

La temperatura del suelo fue estadísticamente inferior $(\mathrm{p}<0.05)$ fuera de la copa de los árboles, habiendo 12.6 y $8.9 \%$ mayor temperatura en épocas lluviosa y seca, respectivamente; mientras que la humedad del suelo fue menor fuera de la copa de los árboles en épocas lluviosa (7.0\%) y seca (34\%) (Cuadro 2). La mayor humedad bajo la copa de los árboles crea un microclima apropiado para el normal crecimiento de la pastura en esta zona, siendo crucial en las épocas de menor precipitación. La mayor humedad del suelo bajo la copa de los árbo-

Cuadro 2. Variables de temperatura y humedad del suelo alrededor de árboles de faique (Acacia macracantha)

\begin{tabular}{lllll}
\hline & \multicolumn{2}{c}{ Temp $\left({ }^{\circ} \mathrm{C}\right)$} & \multicolumn{2}{c}{ Humedad $(\%)$} \\
\hline & Lluvia & Seca & Lluvia & Seca \\
FCA & $28.5^{\mathrm{a}}$ & $33.9^{\mathrm{a}}$ & $15.6^{\mathrm{a}}$ & $10.4^{\mathrm{c}}$ \\
BCAj & $26.5^{\mathrm{b}}$ & $31.3^{\mathrm{b}}$ & $16.9^{\mathrm{ab}}$ & $10.8^{\mathrm{c}}$ \\
BCAm & $24.9^{\mathrm{c}}$ & $30.9^{\mathrm{b}}$ & $16.7^{\mathrm{b}}$ & $11.8^{\mathrm{c}}$ \\
\hline
\end{tabular}

Fuera de la copa del árbol (FCA), bajo la copa del árbol joven (BCAj), bajo la copa del árbol mediano (BCAm) les se debe a la disminución de la evaporación del suelo y la evapotranspiración de la planta (Ludwig et al., 2001).

\section{Tasa de Crecimiento}

La tasa de crecimiento fue significativamente mayor en la época lluviosa (71.1, 58.8 y $51.2 \mathrm{~kg} \mathrm{MS} /$ ha/día fuera de la copa, bajo la copa del árbol joven y mediano, respectivamente) que en la época $(29.6,24.1$ y $19.1 \mathrm{~kg} \mathrm{MS} / \mathrm{ha} /$ día, respectivamente $)(\mathrm{p}<0.05$; Figura 1). La disminución de la tasa de crecimiento bajo la copa de los árboles se debe a que los pastos guinea son de ciclo fotosintético tipo $\mathrm{C}_{4}$, alcanzando su máxima producción con altos niveles de intensidad lumínica (Obispo et al., 2008). Así mismo, el mayor crecimiento fuera de la copa de los árboles está relacionado con la menor calidad del pasto, debido a la rápida acumulación de lignina y celulosa en sus tejidos (Nuñez et al., 2019).

Las tasas de crecimiento encontradas para las dos épocas son superiores a los reportadas por Nuñez (2017) a 30 días de rebrote, quien reporta valores de 46.3, $18.4 \mathrm{y}$ $8.2 \mathrm{~kg} \mathrm{MS} / \mathrm{ha} /$ día en las épocas lluviosa, inicio de lluvias y lluviosa respectivamente, sin considerar la ubicación del pasto respecto a la presencia de árboles. En el trópico peruano (ceja de selva), las tasas de crecimiento y calidad del forraje están sujetas a las precipitaciones pluviales, alcanzando una capacidad de carga de las pasturas de hasta 3.0 UA/ha (Nuñez et al., 2019).

\section{Proteína Cruda}

El porcentaje de PC fue mayor en la época lluviosa en comparación con la época seca $(\mathrm{p}<0.05)$. Así mismo, estos valores se incrementan con la edad del árbol (Cuadro 3), lo cual explica la mejora en la calidad del pasto al mantener el faique en los potreros. Esta mejora significa $3.3 \mathrm{y}+3.1 \%$ de proteína adicional al mantener los árboles medianos (38-49 cm, DAP) en la época lluviosa y seca, respectivamente. 


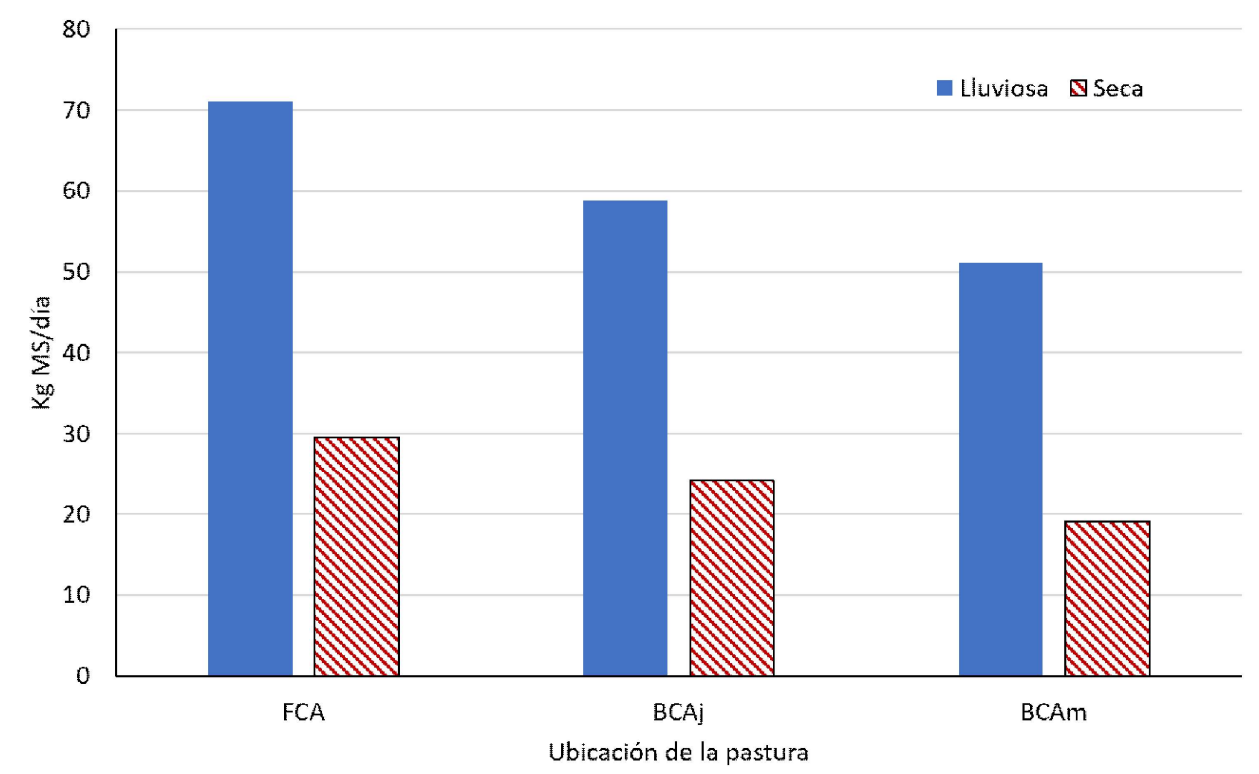

Figura 1. Tasa de crecimiento (kg MS/ha/día) de la pastura Panicum maximum Jacq, en tres ubicaciones: Fuera de la copa del árbol (FCA), bajo la copa del árbol joven (BCAj), bajo la copa del árbol mediano (BCAm)

Cuadro 3. Valores de proteína cruda (\%) del Panicum maximum Jacq. alrededor de árboles de faique (Acacia macracantha)

\begin{tabular}{lccc}
\hline \multirow{2}{*}{ Tratamiento } & \multicolumn{2}{c}{ Época } & \multirow{2}{*}{ Signif. } \\
\cline { 2 - 3 } & Lluviosa & Seca & \\
\hline FCA & $10.58^{\mathrm{c}}$ & $8.93^{\mathrm{d}}$ & 0.0069 \\
BCAj & $12.79^{\mathrm{b}}$ & $10.26^{\mathrm{c}}$ & 0.0005 \\
BCAm & $13.88^{\mathrm{a}}$ & $11.99^{\mathrm{b}}$ & 0.0010 \\
\hline
\end{tabular}

Fuera de la copa del árbol (FCA), bajo la copa del árbol joven (BCAj), bajo la copa del árbol mediano (BCAm)

Los altos contenidos de PC en los pastizales bajo la copa de los arboles podrían estar asociados con un aumento del flujo de nitrógeno en el suelo, especialmente cuando la especie arbórea es una leguminosa que tiene potencial para contribuir a la fijación biológica de N (Xavier et al., 2014); además, los pastos bajo sombra son fisiológicamente más jóvenes que los pastos a pleno sol. En este sentido, Ribaski y Menezes (2002) encontraron una mejora sustancial en PC
$(+16.27 \%)$, al evaluar el valor nutritivo del pasto bufel (Cenchrus ciliaris) bajo la copa del algarrobo (Prosopis juliflora).

\section{Fibra Detergente Neutro (FDN) y Digestibilidad in vitro de la Materia Seca (DIVMS)}

Los valores de FDN Panicum maximum entre tratamientos en la época lluviosa fueron estadísticamente similares, aunque con tendencia a ser menores al incrementar los niveles de sombra. Sin embargo, en la época seca el valor promedio de la FDN disminuyó con la sombra ( $p<0.05$; Cuadro 4$)$. La ligera disminución de FDN registrada en este estudio bajo sombra de los árboles en época 1luviosa podría estar asociada con las paredes celulares más delgadas del esclerénquima en plantas que se cultivan en sombra que aquellas en condiciones de pleno sol (Deinum et al.,1996), ya que estos pastos presentan hojas más delgadas. Como dato comparativo se puede mencionar el trabajo de Paciullo et al. (2016), que en niveles de sombra de 0, 37 y $58 \%$ reportaron valores de $65.2,64.2$ y $64.2 \%$ de FDN. 
Cuadro 4. Valores de fibra detergente neutro (FDN) y digestibilidad in vitro de la materia seca (DIVMS) del Panicum maximum Jacq. alrededor de árboles de faique (Acacia macracantha)

\begin{tabular}{ccccc}
\hline & \multicolumn{2}{c}{ FDN (\%) } & \multicolumn{2}{c}{ DIVMS (\%) } \\
\cline { 2 - 5 } & Lluviosa & Seca & Lluviosa & Seca \\
\hline FCA & $68.97^{\mathrm{a}}$ & $60.96^{\mathrm{c}}$ & $56.44^{\mathrm{b}}$ & $65.06^{\mathrm{a}}$ \\
BCAj & $67.41^{\mathrm{a}}$ & $63.88^{\mathrm{b}}$ & $57.54^{\mathrm{ab}}$ & $60.61^{\mathrm{ab}}$ \\
BCAm & $66.28^{\mathrm{a}}$ & $64.31^{\mathrm{b}}$ & $59.58^{\mathrm{ab}}$ & $61.53^{\mathrm{ab}}$ \\
\hline
\end{tabular}

Fuera de la copa del árbol (FCA), bajo la copa del árbol joven (BCAj), bajo la copa del árbol mediano (BCAm)

$a, b, c$ Letras diferentes dentro de columnas indican diferencias significativas $(p<0.05)$

La DIVMS no presentó diferencias estadísticas entre tratamientos, aunque los valores fueron ligeramente superiores bajo la copa de los árboles en la época lluviosa, coincidiendo con el estudio de Paciullo et al. (2011). Por su parte, Ribaski y Menezes (2002), indican que en el pasto bufel (Cenchrus ciliaris), la DIVMS llega a 44.1\% cuando se encuentra próximo al tronco del algarrobo (Prosopis juliflora), en comparación con el $41.1 \%$ obtenido fuera de la copa del árbol.

\section{Calcio y Fósforo}

El contenido de calcio en el pasto presentó diferencias estadísticas $(\mathrm{p}<0.05)$ entre tratamientos, alcanzando mayores valores bajo la copa del árbol joven $(0.83 \%)$ y bajo la copa del árbol mediano $(0.92 \%)$ en la época lluviosa (Cuadro 5). En la época seca se presentó la misma tendencia, pero sin diferencia estadística significativa. Por su parte, Ribaski y Menezes (2002) reportan tendencias similares bajo y fuera de la copa del árbol (2.1 y $1.9 \%$, respectivamente en el pasto bufel (Cenchrus ciliaris) bajo la copa del algarrobo (Prosopis juliflora). Este comportamiento se debe probablemente por la capacidad de la especie arbórea (raíz pivotante y profunda) para extraer el calcio desde la parte más profunda, sumado al reciclaje de la hojarasca que es aprovechada por la pastura (Sadeghian et al., 1998; Romero 2018).

La cantidad de fósforo en la pastura presentó diferencias estadísticas $(\mathrm{p}<0.05)$ para el caso de fuera de la copa del árbol $(0.19 \%)$ y bajo la copa del árbol mediano $(0.24 \%)$ en la época lluviosa, mientras que mostró similar tendencia en la época seca, pero sin diferencias significativas (Cuadro 5).

Cuadro 5. Valores de calcio y fosforo del Panicum maximum Jacq. alrededor de árboles de faique (Acacia macracantha)

\begin{tabular}{ccccc}
\hline & \multicolumn{2}{c}{ Calcio (\%) } & \multicolumn{2}{c}{ Fósforo (\%) } \\
\cline { 2 - 5 } & Lluviosa & Seca & Lluviosa & Seca \\
\hline FCA & $0.72^{\mathrm{c}}$ & $0.73^{\mathrm{c}}$ & $0.19^{\mathrm{b}}$ & $0.088^{\mathrm{c}}$ \\
BCAj & $0.83^{\mathrm{b}}$ & $0.87^{\mathrm{b}}$ & $0.21^{\mathrm{b}}$ & $0.097^{\mathrm{c}}$ \\
BCAm & $0.91^{\mathrm{b}}$ & $1.06^{\mathrm{a}}$ & $0.24^{\mathrm{a}}$ & $0.098^{\mathrm{c}}$ \\
\hline
\end{tabular}

Fuera de la copa del árbol (FCA), bajo la copa del árbol joven (BCAj), bajo la copa del árbol mediano (BCAm)

$a, b, c$ Letras diferentes dentro de variables indican diferencias significativas $(p<0.05)$

La menor disponibilidad del fósforo en la pastura en época seca se debe a su poca disponibilidad en el suelo (Cuadro 1), sumado a que el agua constituye un vehículo natural de transporte (difusión) del fósforo en el suelo. La mayor disponibilidad (flujo) estacional (época lluviosa) favorece la absorción por la pastura (Casanova y Eduardo, 2005). Similar comportamiento estacional en este sistema silvopastoril fue encontrado por Núñez (2017). El fósforo es un mineral importante en la alimentación de los rumiantes; sin embargo, su concentración en el pasto puede estar alrededor de $0.2 \%$ (Tejos, 2001). 


\section{Conclusiones}

- La presencia de árboles dentro de los potreros mejoró el contenido nutricional de la pastura en términos de proteína $(+3.3 \mathrm{y}+3.06 \%)$, calcio $(+0.03 \mathrm{y}+0.1 \%)$ $\mathrm{y}$ fósforo $(+0.19 \mathrm{y}+0.33 \%)$ en época lluviosa y seca, respectivamente.

- La presencia de árboles dentro de los potreros mejoró el porcentaje de nutrientes (calcio: $+30.7 \%$ y fósforo: $+37.69 \%$ ) disponibles en el suelo, aumentando su fertilidad; lo cual se reflejó en la mejor calidad nutricional de la pastura.

\section{Agradecimientos}

Al Laboratorio de Ecología y Utilización de Pastizales, Universidad Nacional Agraria La Molina, y a la Empresa Agropecuaria San Antonio, Jaén, Cajamarca.

\section{Literatura Citada}

1. Abraham EM, Kyriazopoulos AP, Parissi ZM, Kostopoulou P, Karatassiou M, Anjalanido K, Katsouta $C$. 2014. Growth, dry matter production, phenotypic plasticity, and nutritive value of three natural popula-tions of Dactylis glomerata $\mathrm{L}$ under various shading treatments. Agroforest Syst 88: 287-299. doi: 10.1007/ s10457-014-9682-9

2. Alegre JC, Vega R, La Torre B. 2012. Manual de manejo de suelos con sistemas silvopastoriles.Lima, Perú: Univ. Nacional Agraria La Molina.

3. [AOAC] Association of Official Analytical Chemist. 2016. Official Methods of Analysis. 975.03 Cap. 3. USA: AOAC.

4. [ASRM] American Society of Range Management and Agriculture Board. 1962. Basic problems and techniques in range research. Pub. $\mathrm{N}^{\circ}$. 890.Washington DC, USA: National Academy of Sciences. $336 \mathrm{p}$.
5. Casanova O, Eduardo F. 2005. Introducción a la ciencia del suelo. Caracas: UCV Consejo de Desarrollo Científico y Humanístico. 482 p.

6. Deinum B, Sulastri RD, Zeinab MHJ, Maassen A. 1996. Effects of light intensity on growth, anatomy and forage quality of two tropical grasses (Brachiaria brizantha and Panicum maximum var. trichoglume). Netherlands J Agric Sci 44: 111-124.

7. [FAO] Organización de la Naciones Unidas para la Agricultura y la Alimentación. 2004. Inventario forestal nacional. Manual de campo modelo. Guatemala. [Internet]. Disponible en: http:// www.fao.org/3/a-ae578s.pdf

8. Holdridge L. 2000. Ecología basada en zonas de vida. San José, Costa Rica: IICA. 216 p.

9. García I. 2002. Nutrición de rumiantes. [Internet]. Disponible en: http:// www.angelfire.com/ar/iagg101/images/ vansoest2.PDF

10. Geenty KG, Rattray PV. 1987. The energy requirements of grazing sheep and cattle. Livestock feeding on pasture. New Zealand Society of Animal Production. Occasional Publication 10: 39-53.

11. Ledesma R. 2006. Desarrollo de sistemas ganaderos: una alternativa de manejo en ecosistemas degradados del Chaco semiárido. Tesis de Maestría. Argentina: Univ. Nacional de Santiago del Estero. $134 \mathrm{p}$.

12. Ludwig F, De Kroon H, Prins $H$, Berendse F. 2001. Effects of nutrients and shade on tree grass interaction in an East African savanna. J Veg Sci 12: 579588. doi: $10.2307 / 3237009$

13. Martínez M. 1971. Calibración y comparación de algunos métodos para la determinación de humedad del suelo. Tesis de Maestría. Chapingo, México: Colegio de Postgraduados. $120 \mathrm{p}$.

14. Navarro G 2013. Química agrícola Química del suelo y de los nutrientes esenciales para las plantas. $3^{\circ}$ ed. España: Mundi-Prensa. 508 p. 
15. NuñezJ. 2017. Perfil alimentario y plan de pastoreo para la producción lechera con pasturas panicum maximum Jacq. Tesis de Maestría. Lima, Perú: Univ. Nacional Agraria La Molina. 112 p.

16. Núñez Delgado J, Naupari Vasquez J, Flores Mariazza E. 2019. Comportamiento nutricional y perfil alimentario de la producción lechera en pastos cultivados (Panicum maximum Jacq). Rev Inv Vet Perú 30: 178-192. doi: 10.15381/ rivep.v30i1.15681

17. Obispo N, Espinoza Y, Gil, J, Ovalles F, Rodriguez, M. 2008. Efecto del sombreado sobre la producción y calidad del pasto guinea (Panicum maximum) en un sistema silvopastoril.Zootec Trop 26:285-288.

18. Oliva S. 2016. Influencia de factores socioeconómicos y ambientales sobre la adopción de tecnologías silvopastoriles por productores ganaderos, Distrito de Molinopampa, Amazonas. Tesis de Maestría. Lima, Perú: Univ. Nacional Agraria La Molina. $102 \mathrm{p}$.

19. Paciullo D, de Castro C, de Miranda Gomide C, Maurício R, Pires M, Müller M, Xavier D. 2011. Performance of dairy heifers in a silvopastoral system. Livestock Sci 141: 166-172. doi: 10.1016/j.livsci.2011.05.012

20. Paciullo D, Gomide C, Castro C, Mauricio R, Fernandes P, Morenz M. 2016. Morphogenesis, biomass and nutritive value of Panicum maximum under different shade levels and fertilizer nitrogen rates. Grass Forage Sci. doi: 10.1111/gfs. 12264

21. Ribaski J, Menezes E. 2002. Disponibilidad y calidad del pasto buffel (Cenchrus ciliaris) en un sistema silvopastoril con algarrobo (Prosopis juliflora) en la región semi-árida brasileña. Agroforestería Américas 9: 8-18.

22. Romero G. 2018. Estado nutricional del pasto guinea (Panicum maximun Jacq.) asociado con faique (Acacia macracan- tha) en un sistema silvopas-toril en JaénCajamarca. Tesis de Maestría. Lima, Perú: Univ. Nacional Agraria La Molina. 69 p.

23. Sadeghian S, Rivera L, Gomez M. 1998. Impacto de sistemas de Idilladerfa sobre las características físicas, químicas y biológicas de suelos en los Andes de Colombia. In: Conferencia Electrónica de la FAO sobre «Agroforestería para la producción animal en Latinoamérica tropical». [Internet]. Disponible en: http:/ /www.fao.org/ag/aga/agap/frg/agrofor1/ Agrofor1.htm

24. [SENAMHI] Servicio Nacional de Meteorología e Hidrología del Perú. 2018. Ministerio del Ambiente. [Internet]. Disponible en: https:// www.senamhi.gob.pe

25. Tejos R. 2001. Alternativas de manejo de pasturas para bovinos post destete. En: XII Jornadas Técnicas de Ganadería en el estado de Táchira. Venezuela.

26. Tilley M, Terry A. 1963. Two stage technique for the in vitro digestion of forage crops. Grass Forage Sci 18: 104111. doi: $10.1111 / \mathrm{j} .1365-2494.1963 .-$ tb00335.x

27. Van Soest P. 1985. Composition, fiber quality and nutritive value of forages. In: Forages. The Science of Grassland and Agriculture. Ames, USA: Iowa State University Press. p 412-444.

28. Wilson J, Ludlow M. 1991. The environment and potencial growth of herbage under plantations. In: Shelton HM, Stur WW (eds). Forages for plantations crops. Canberra, Australia. ACIAR Proc 32.

29. Xavier DF, Ledo FJ, Paciullo DS, Urquiaga S, Alves BJ, Bodde RM. 2014. Nitrogen cycling in a Brachiariabased silvopastoral system in the Atlantic forest region of Minas Gerais, Brazil. Nutr Cycl Agroecosys 99: 45-62. doi: 10.1007/s10705-014-961 